\title{
Colorectal cancer screening in patients with spinal cord injury yields similar results to the general population with an effective bowel preparation: a retrospective chart audit
}

\author{
Brandon J. Teng ${ }^{1} \cdot$ Shawn H. Song ${ }^{1,2} \cdot$ Jelena N. Svircev ${ }^{1,2} \cdot$ Jason A. Dominitz ${ }^{1,2} \cdot$ Stephen P. Burns $^{1,2}$
}

Received: 11 July 2017 / Revised: 18 September 2017 / Accepted: 28 September 2017 / Published online: 24 November 2017

(c) International Spinal Cord Society 2018

\begin{abstract}
Study design Retrospective chart audit.

Objectives To compare adequacy of colonoscopy bowel preparation and diagnostic findings between persons with SCI receiving an extended inpatient bowel preparation and the general population.

Setting Veterans Affairs Puget Sound Healthcare System, Seattle, WA, USA.

Methods We reviewed an electronic database of all colonoscopies performed at a tertiary Veterans Affairs medical center between 7/12/13 and 15/10/15. Patients with SCI received a multi-day bowel preparation with magnesium citrate, and 8-10 liters of polyethylene glycol-3350 and electrolyte colonic lavage solution (PEG-ELS) over two and one half days. The control population received a standard bowel preparation consisting of magnesium citrate and 4 liters of PEG-ELS over 1 day.

Results Two hundred and fifty-five patients were included in the study, including 85 patients with SCI. Average risk screening was a more common colonoscopy indication in patients with SCI vs. the control population ( $24 \mathrm{vs.} 13 \% p=0.03$ ). There was no difference in adequacy of bowel preparation $(87 \mathrm{vs.} 85 \%, p=0.73)$ or adenoma detection rate $(55$ vs. $51 \%$, $p=0.59$ ) when comparing patients with SCI with the control population. No difference in polyp histopathology was detected $(p=0.748)$.

Conclusions Our study demonstrated that an extended bowel preparation for patients with SCI produces similar bowel preparation results and diagnostic yield when compared to patients without SCI undergoing colonoscopy.
\end{abstract}

\section{Introduction}

Colorectal cancer (CRC) remains the second leading cause of cancer death in the United States [1]. Although many different screening tests are available, colonoscopy is the most widely used and likely most effective. Colonoscopy is required for diagnostic evaluation of non-colonoscopic screening tests, such as positive fecal immunochemical test (FIT) [2]. The ability of colonoscopy to detect adenomas, however, is thought to be dependent upon the quality

Brandon J. Teng

bteng@uw.edu

University of Washington, Seattle, WA, USA

2 Veterans Affairs Puget Sound Health Care System, Seattle, WA, USA of bowel preparation, with inadequate bowel cleansing resulting in incomplete visualization of polyps. This has particular relevance among patients with spinal cord injury (SCI), a population in which preventative screening tests, such as colonoscopy are gaining more attention due to the improvement in life expectancy $[3,4]$.

There are several unique challenges in performing colonoscopy in patients with SCI. First, the administration of a bowel preparation can present logistical challenges for the person and/or their caregiver, such as frequent bathroom transfers and clean-up due to voluminous stools. In addition, prolonged commode sitting or transfers may place neurogenic skin at high risk of breakdown. Nausea and abdominal bloating/discomfort are potential triggers for autonomic dysreflexia in patients with SCI above the T6 level. Finally, decreased colonic motility places patients with SCI at higher risk for inadequate bowel cleansing with standard preparations [5-8]. A single center study reported 
adequate bowel preparation in only $25 \%$ of colonoscopies in a SCI population when using a standard one-day preparation, much lower than the $85 \%$ benchmark established by the gastroenterology community [9]. Not surprisingly, the adenoma detection rate (ADR, measured as the proportion of screening colonoscopies in which an adenomatous polyp is found) has been reported to be lower in patients with SCI [5], although whether mortality is increased due to inadequate screening has not been proven $[5,10]$. There is also an associated increased burden of colonoscopy on the SCI population due to the cost of repeat colonoscopies from aborted and repeated examinations [11].

A recent study by our group demonstrated that an extended bowel preparation is both safe and effective for patients with SCI, with $89 \%$ of patients having adequate bowel cleansing at colonoscopy and without significant electrolyte abnormalities [12]. We did not, however, compare the ADR among these patients to patients without SCI who underwent standard bowel preparation. In addition, we did not compare the pathologic findings among patients with SCI to those without SCI, a relevant question given a previous report that colorectal cancer may be more advanced upon presentation among patients with SCI [13] although this has been disputed [14]. Incomplete bowel preparation leading to incomplete visualization during colonoscopy is thought to be the primary cause of this low ADR [5], as poor bowel preparation in the general population is associated with missed neoplastic lesions [15].

\section{Objective}

We aimed to determine whether the extended bowel preparation among patients with SCI produces similar efficacy when compared to a standard bowel preparation among patients without SCI, whether the ADR differs between SCI and non-SCI patients undergoing colonoscopy, and whether there were any differences in pathologic findings between SCI and non-SCI patients.

\section{Methods}

\section{Study design}

All patients with SCI who underwent colonoscopy at the Seattle, Washington, campus of the VA Puget Sound Health Care System (VAPSHCS) from 07/12/13 to $15 / 10 / 15$ were identified from the endoscopy unit database crossreferenced with a patient registry maintained by the SCI Service at VAPSHCS; this group was comprised of patients with SCI and included the 53 patients with SCI reported in our prior study plus additional subjects [12]. A control group of patients without SCI who underwent colonoscopy at the same site was randomly selected from the endoscopy unit database from the same time period with 2:1 oversampling of these control subjects. Potential patients were excluded if the colonoscopy was aborted early. The electronic medical record was used to identify demographics, indication for colonoscopy, adequacy of bowel preparation, study findings, and pathology findings, and for patients with SCI the neurological classification of their SCI.

\section{Bowel preparation methodology}

Nearly the entire group of patients with SCI received a bowel preparation protocol requiring an inpatient admission with 2-2.5 days of bowel preparation with polyethylene glycol-3350 and electrolyte colonic lavage solution (PEG-ELS). Per this protocol, patients with SCI receiving inpatient colonoscopies were placed on clear liquid diets and given $480 \mathrm{ml}$ of magnesium citrate the evening 3 days prior to the colonoscopy. Two days before the scheduled procedure 4 liters of PEG-ELS was administered over a 2-h period, and repeated again the following day. Two more liters of PEG-ELS was administered if rectal/colostomy output was not clear on the morning of colonoscopy. All patients continued their home bowel care program during the process, including self-administered or nursing-assisted rectal digital stimulation, suppository insertion, and/or mini-enema administration. The control group received 1 day of bowel preparation determined by the gastroenterologist performing the study. The standard bowel preparation for the control group consisted of $480 \mathrm{ml}$ of magnesium citrate on the afternoon prior to colonoscopy followed by 41 PEG-ELS administered either the evening prior to colonoscopy or split between the evening and the morning of the colonoscopy. Nearly all studies for the control group were performed in an outpatient setting.

\section{Colonoscopy}

All colonoscopies were performed at the VA Puget Sound Health Care System either directly by or under the supervision of a board certified gastroenterologist. Patients were classified to have an adequate bowel preparation if they were graded "excellent" or "good" on the Aronchick scale or if they had a Boston Bowel Prep Score (BPPS) of greater than or equal to 2 in all three segments of the colon [16, 17]. Indications for colonoscopy were categorized into average risk screening, high-risk screening, surveillance of inflammatory bowel disease, evaluation of a positive FIT, 
Table 1 Subject demographics

\begin{tabular}{lcll}
\hline Subject demographics & $\begin{array}{l}\text { SCI } \\
(n=85)\end{array}$ & $\begin{array}{l}\text { Control } \\
(n=170)\end{array}$ & $p$-value \\
\hline Male $(n, \%)$ & $85(100 \%)$ & $156(92 \%)$ & 0.006 \\
Age in years (mean, SD) & $63.3(7.2)$ & $61.2(10.7)$ & 0.023 \\
Injury duration in years & $20.6(14.6)$ & NA & \\
(mean, SD) & & & \\
Neurological level of Injury $(n, \%)$ & & \\
$\quad$ Cervical & $53(62 \%)$ & NA & \\
$\quad$ Thoracic & $29(34 \%)$ & & \\
$\quad$ Lumbar & $3(4 \%)$ & & \\
ASIA Impairment Scale $(n, \%)$ & & \\
AIS A & $35(41 \%)$ & NA & \\
AIS B & $7(8 \%)$ & & \\
AIS C & $7(8 \%)$ & & \\
AIS D & $36(42 \%)$ & &
\end{tabular}

$S C I$ spinal cord injury, $S D$ standard deviation, ASIA American Spinal Injury Association, AIS ASIA Impairment Scale, $N A$ not applicable

evaluation of gastrointestinal bleeding (e.g. minor rectal bleeding) or other indications. Pathologic findings were categorized into no polyps, hyperplastic polyp, adenoma or adenocarcinoma based upon the most advanced finding. The designation of adenoma included villous and sessile serrated adenoma.

\section{Statistical analysis}

Data are expressed as mean \pm standard deviation. The Fisher's exact test or Chi-square test was used to compare statistical differences in completion of bowel preparation, adequacy of bowel preparation, and study indication. All statistical analyses were performed with SPSS for Windows. A $p$-value of $<0.05$ was considered statistically significant.

\section{Results}

\section{Baseline data}

The SCI patient group included 85 patients who underwent colonoscopy; 77 (91\%) were performed as inpatient studies with the 2-2.5-day bowel preparation, and all were male. The control group included 170 patients, 14 of whom were female. Average age was slightly higher for the SCI cohort than for controls (63 vs. 61 years, $p=0.023$ ) (Table 1). The majority $(62 \%)$ of the SCI cohort had tetraplegia. The degree of neurological impairment per the American Spinal Injury Association Impairment Scale (AIS) was AIS A for $35(41 \%)$, AIS B for $7(8 \%)$, AIS C for $7(8 \%)$ and AIS D for $36(42 \%)$.
Table 2 Colonoscopy indication

\begin{tabular}{llll}
\hline Colonoscopy indication $(n, \%)$ & SCI & Control & $p$-value \\
\hline Average risk screening & $21(25 \%)$ & $23(14 \%)$ & 0.03 \\
High risk screening & $1(1 \%)$ & $11(7 \%)$ & \\
Adenoma surveillance & $33(39 \%)$ & $49(29 \%)$ & \\
Inflammatory bowel disease & $1(1 \%)$ & $10(6 \%)$ \\
surveillance & & \\
Diagnostic: FIT positive & $10(12 \%)$ & $20(12 \%)$ \\
Diagnostic: Gastrointestinal bleed & $11(13 \%)$ & $15(9 \%)$ \\
Diagnostic: Other & $8(9 \%)$ & $42(25 \%)$ & \\
\hline
\end{tabular}

SCI spinal cord injury, FIT fecal immunohistochemical test

\section{Outcome results}

Compared to the control group, the SCI patient group was more likely to receive a colonoscopy for average risk screening (25 vs. 14\%), for surveillance after prior adenomatous polyps (39 vs. 29\%), and for evaluation of gastrointestinal bleeding (13 vs. 9\%) including positive FIT screening (Table 2). A smaller proportion of the SCI patient group were recorded as having completed the prescribed cathartic regimen (68 vs. $81 \%, p<0.00001$ ), although there was no difference in adequacy of bowel preparation $(87 \%$ of SCI vs. $85 \%$ of control, difference of $2 \%$ (95\% CI -9 to $11 \%)$ ) (Table 3). There was no difference in ADR between the SCI patient and control group (55 vs. $51 \%$, difference of $4 \%(95 \% \mathrm{CI}-10$ to $17 \%))$, and no difference in polyp findings $(p=0.748)$ (Table 4$)$.

\section{Discussion}

High-quality bowel preparation is required for colonoscopy to detect and prevent CRC. Our previous study demonstrated that an extensive bowel preparation regimen for patients with SCI is safe and resulted in adequate bowel cleansing in a high percentage of patients. The current study compares the efficacy of the extended bowel preparation among patients with SCI to the standard bowel preparation among patients without SCI. In addition, this study compares the ADR and polyp findings between patients with and without SCI.

The SCI-specific bowel preparation resulted in similar preparation adequacy compared to the control group. For patients with SCI who underwent the inpatient preparation, $87 \%$ demonstrated adequate bowel preparation compared with $85 \%$ of our control population (Table 3 ). There was no variation in preparation adequacy by segment of colon. Both populations met the recent US Multisociety Task Force recommended benchmark for bowel preparation 
Table 3 Bowel preparation and adequancy

\begin{tabular}{|c|c|c|c|c|}
\hline Bowel preparation & SCI & Control & $p$-value & $\begin{array}{l}\text { Between-group difference (95\% } \\
\text { confidence interval) }\end{array}$ \\
\hline \multicolumn{5}{|c|}{ Tolerance of full preparation $(n, \%)$} \\
\hline Completed & $58(68 \%)$ & $138(81 \%)$ & $<0.00001$ & NA \\
\hline Incomplete & $17(20 \%)$ & $2(1 \%)$ & & \\
\hline Unknown & $10(12 \%)$ & $30(18 \%)$ & & \\
\hline \multicolumn{5}{|l|}{ Adequacy of preparation } \\
\hline Adequate $^{\mathrm{a}}(n, \%)$ & $74(87 \%)$ & $145(85 \%)$ & 0.70 & $2 \%(-9$ to $11 \%)$ \\
\hline $\begin{array}{l}\text { BBPS total score } \\
\text { (mean, SD) }\end{array}$ & $7.1(1.5)$ & $7.6(1.8)$ & 0.10 & $-0.5(-0.9$ to 0.08$)$ \\
\hline $\begin{array}{l}\text { BBPS right (mean, } \\
\text { SD) }\end{array}$ & $2.4(0.6)$ & $2.6(0.6)$ & 0.06 & $-0.2(-0.4$ to 0.006$)$ \\
\hline $\begin{array}{l}\text { BBPS transverse } \\
(\text { mean, SD) }\end{array}$ & $2.6(0.6)$ & $2.6(0.6)$ & 0.71 & $-0.03(-0.2$ to 0.2$)$ \\
\hline BBPS left (mean, SD) & $2.2(0.7)$ & $2.4(0.8)$ & 0.14 & $-0.2(-0.4$ to 0.05$)$ \\
\hline Aronchick scores $^{\mathrm{c}}(n)$ & $\begin{array}{l}\text { Excellent: } 22 \\
\text { Good: } 25 \\
\text { Fair: } 6 \\
\text { Poor: } 2\end{array}$ & $\begin{array}{l}\text { Excellent: } 19 \\
\text { Good: } 24 \\
\text { Fair: } 6 \\
\text { Poor: } 8\end{array}$ & 0.28 & NA \\
\hline
\end{tabular}

SCI spinal cord injury, BBPS Boston Bowel Preparation Score, SD standard deviation, OR odds ratio, 95\% CI 95\% confidence interval, NA not applicable

aPatients were classified to have an adequate bowel preparation if they were graded "excellent" or "good" on the Aronchick scale or if they had a Boston Bowel Prep Score (BPPS) of greater than or equal to 2 in all three segments of the colon

${ }^{\mathrm{b}}$ Determined for 54 SCI patients and 142 control patients

${ }^{c}$ Determined for 55 SCI patients and 57 control patients adequacy [9]. The lower rate of tolerance of the full preparation in the SCI patient group could indicate difficulty tolerating the volume of the preparation, but could reflect differential reporting of preparation tolerance, since SCI nursing staff reported tolerance for most SCI subjects while control subjects self-reported.

Prior studies demonstrated that the use of standard bowel preparation for patients with SCI resulted in lower ADR than in a control population [5, 18]. Adequate bowel preparation is associated with higher ADR [16], and our study showed no difference between ADR between patients with $\mathrm{SCI}$ and the control group (55 vs. $51 \%, p=0.59)$. Though ADR was originally intended to be used only for comparing results of screening colonoscopies [11], we still found no difference in ADR between the SCI patient group and control group when analyzed by indication (Table 4). This finding supports that the extended bowel preparation protocol is achieving comparable clinical benefit for patients with SCI to that seen in the control group. Given previous evidence that patients with SCI receive fewer preventativescreening tests, there is a theoretical concern that adenocarcinoma may be found with greater frequency in this population. A previous study suggested that the diagnosis of CRC was made later in the natural history of the disease compared to non-SCI patients $[13,19]$. Though we found no significant difference in frequency of adenocarcinoma between the SCI and non-SCI groups, our sample size may be too small to reliably evaluate this outcome. However, there was no significant difference in the detection rates of hyperplastic polyps, adenoma, or adenocarcinoma between the two populations.

Our study does have some additional limitations. First, this was a single center study at a tertiary care Veterans Affairs Medical Center with a dedicated SCI unit and findings may not be generalizable to all settings. In addition to the specialized bowel preparation protocol, patients with SCI were cared for by nurses and staff trained in digital rectal stimulation, maintaining skin integrity and managing voluminous stool output. These resources may not be available to patients with SCI who are not Veterans, and the extensive bowel preparation protocol is likely difficult to perform at home or in other settings. Another limitation of our study is the reliance on retrospectively collected, for which we cannot ensure complete accuracy. Both SCI and control patient groups fail to include any individuals for whom preparation intolerance was so severe that colonoscopy was not attempted, and it is possible that this could have occurred to a greater degree with the extended bowel preparation. Finally, though there were no statistically significant $p$-values when comparing bowel preparation 
Table 4 Adenoma detection rate and polyp findings

\begin{tabular}{|c|c|c|c|c|}
\hline Adenoma detection $(n, \%)$ & SCI & Control & $p$-value & $\begin{array}{l}\text { Between-group difference } \\
\text { ( } 95 \% \text { confidence interval) }\end{array}$ \\
\hline Overall & $47(55 \%)$ & $88(51 \%)$ & 0.59 & $4 \%(-10$ to $17 \%)$ \\
\hline \multicolumn{5}{|l|}{ By indication } \\
\hline Average risk screening & $\begin{array}{l}12 / 21 \\
(57 \%)\end{array}$ & $14 / 23(60 \%)$ & 0.80 & $-4 \%(-33$ to $26 \%)$ \\
\hline High risk/surveillance & $\begin{array}{l}12 / 35 \\
(34 \%)\end{array}$ & $32 / 70(45 \%)$ & 0.30 & $-11 \%(-30$ to $10 \%)$ \\
\hline Diagnostic & $\begin{array}{l}14 / 29 \\
(48 \%)\end{array}$ & $36 / 77(47 \%)$ & 1.0 & $2 \%(-20$ to $23 \%)$ \\
\hline \multicolumn{5}{|l|}{ Polyp findings } \\
\hline No polyp & $29(34 \%)$ & $62(36 \%)$ & 0.75 & NA \\
\hline Hyperplastic & $9(10 \%)$ & $20(12 \%)$ & & \\
\hline Adenoma & $46(54 \%)$ & $83(49 \%)$ & & \\
\hline Adenocarcinoma & $1(1 \%)$ & $5(3 \%)$ & & \\
\hline
\end{tabular}

$S C I$ spinal cord injury, NA not applicable adequacy and diagnostic yield between patients with and without SCI, the $95 \%$ confidence intervals of the betweengroup differences were relatively large. However, even the lower limit of the $95 \%$ confidence interval of the betweengroup difference in bowel preparation adequacy (9\% lower in patients with SCI compared to control patients) represents a clinically meaningful improvement in precolonoscopy bowel preparation in the population with SCI [9]. Similarly, the lower limit of the $95 \%$ confidence interval of the between-group difference in ADR (10\% lower in patient with SCI compared to control patients) still represents an improvement in ADR compared to previously published studies [5].

There are notable strengths with our study. Other centers have studied novel bowel preparation for patients with SCI, including sodium phosphosoda + PEG-ELS [18], pulsed irrigation-enhanced evacuation [20], and addition of neostigmine to a standard preparation [21]. Of these regimens, only the addition of neostigmine to a standard preparation demonstrated a comparable bowel preparatory rating and ADR. However our study had a larger number of subjects with SCI, produced a high rate of adequate preparation, with a higher proportion of procedures performed for screening and polyp surveillance [12, 21]. Our protocol may also be more widely available to patients as the usage of neostigmine often requires more intensive monitoring and excludes patients with cardiac or obstructive pulmonary disease due to the risks of the medication. This supports the use of our bowel preparation for detection and prevention of CRC.

\section{Conclusions}

In conclusion, our study demonstrated that an extended bowel preparation for patients with SCI produces similar bowel preparation results, adenoma detection rate, and diagnostic yield when compared to non-SCI subjects undergoing colonoscopy. Perhaps the largest and most robust study to date, our protocol appears to be safe and effective for patients with SCI who need colonoscopy.

Acknowledgements The manuscript represents original work and has not been submitted for acceptance for publication in any other journal. This material is the result of work supported by Veterans Affairs Puget Sound Health Care System (Seattle, WA). The contents of this work do not represent the views of the U.S. Department of Veterans Affairs or the United States Government.

\section{Compliance with ethical standards}

Conflict of interest The authors declare they have no competing interests.

Ethical approval No ethics approval was required. Study was approved by the VA Puget Sound IRB. This material is the result of work supported by Veterans Affairs Puget Sound Health Care System (Seattle, WA). The contents of this work do not represent the views of the US Department of Veterans Affairs or the United States Government.

\section{References}

1. Siegel RL, Miller KD, Jemal A. Cancer statistics, 2017. Cancer J Clin 2017;67:7-30.

2. Doubeni $\mathrm{C}$, et al. Effectiveness of screening colonoscopy in reducing the risk of death from right and left colon cancer: a large community-based study. Gut. 2016. doi: 10.1136/gutjnl-2016-312712.

3. Shavelle R, DeVivo M, Brooks J, Strauss D, Paculdo D. Improvements in long-term survival after spinal cord injury? Arch Phys Med Rehabil 2015;96:645-51.

4. Johnston MV, Diab ME, Chu BC, Kirshblum S. Preventive services and health behaviors among people with spinal cord injury. J Spinal Cord Med 2005;28:43-54. 
5. Morris BP, Kucchal T, Burgess AN. Colonoscopy after spinal cord injury: a case-control study. Spinal Cord 2015;53:32-5.

6. Fajardo NR, et al. Decreased colonic motility in persons with chronic spinal cord injury. Am J Gastroenterol 2003;98:128-34.

7. Singal AK, Rosman AS, Bauman WA, Korsten MA. Recent concepts in the management of bowel problems after spinal cord injury. Adv Med Sci 2006;51:15-22.

8. Calder KB, Estores IM, Krassioukov A. Autonomic dysreflexia and associated acute neurogenic pulmonary edema in a patient with spinal cord injury: a case report and review of the literature. Spinal Cord 2009;47:423-5.

9. Johnson DA, et al. Optimizing adequacy of bowel cleansing for colonoscopy: recommendations from the US multi-society task force on colorectal cancer. Gastroenterology 2014;147:903-24.

10. Han SJ, Kim CM, Lee JE, Lee TH. Colonoscopic lesions in patients with spinal cord injury. J Spinal Cord Med 2009;32:404-7.

11. Rex DK, Imperiale TF, Latinovich DR, Bratcher LL. Impact of bowel preparation on efficiency and cost of colonoscopy. Am J Gastroenterol 2002;97:1696-700.

12. Song S, Svircev J, Teng B, Dominitz J, Burns S. A safe and effective multi-day colonoscopy bowel preparation for individuals with spinal cord injuries. J Spinal Cord Med 2017;1:1-2-8.

13. Frisbie J, Chopra S, Foo D, Sarkarati M. Colorectal carcinoma and myelopathy. J Am Parapleg Soc 1984;7:33-4-6.
14. Kao C, et al. Risk of nongenitourinary cancers in patients with spinal cord injury: a population-based cohort study. Medicine 2016;95:e2462.

15. Harewood GC, Sharma VK, de Garmo P. Impact of colonoscopy preparation quality on detection of suspected colonic neoplasia. Gastrointest Endosc 2003;58:76-9.

16. Clark B, et al. Quantification of adequate bowel preparation for screening or surveillance colonoscopy in men. Gastroenterology 2016;150:396-405.

17. Bechtold M, Mir F, Puli S, Nguyen D. Optimizing bowel preparation for colonoscopy: a guide to enhance quality of visualization. Ann Gastroenterol 2016;29:137-46.

18. Ancha HR, et al. Clinical trial: the efficacy and safety of routine bowel cleansing agents for elective colonoscopy in persons with spinal cord injury-a randomized prospective single-blind study. Aliment Pharmacol Ther 2009;30:1110-7.

19. Stratton MD, et al. Colorectal cancer in patients with previous spinal cord injury. Dis Colon Rectum 1996;39:965-8.

20. Lyons B, et al. Comparison between pulsed irrigation enhanced evacuation and polyethylene glycol-electrolyte lavage solution for bowel preparation prior to elective colonoscopy in veterans with spinal cord injury. J Spinal Cord Med 2015;38:805-05-11.

21. Korsten MA, et al. Neostigmine administered with moviprep improves bowel preparation for elective colonoscopy in patients with spinal cord injury: a randomized study. J Clin Gastroenterol. 2015;49:751-6. 